\title{
Evaluation of the uptake of bioactive components from wheat-bran and wheat-aleurone fractions in healthy adults
}

\author{
E. M. Keaveney ${ }^{1}$, L. L. Hamill ${ }^{1}$, R. K. Price ${ }^{1}, J$ M. W. Wallace ${ }^{1}$, H. McNulty $^{1}$, M. Ward ${ }^{1}$, \\ J. J. Strain ${ }^{1}$, P. M. Ueland ${ }^{2}$, J. M. Scott ${ }^{3}$, A. M. Molloy ${ }^{3}$ and R. W. Welch ${ }^{1}$ \\ ${ }^{1}$ Northern Ireland Centre for Food and Health (NICHE), University of Ulster, Coleraine BT52 ISA, UK, ${ }^{2}$ Bevital, University \\ of Bergen, Bergen, Norway and ${ }^{3}$ Department of Biochemistry, Trinity College Dublin, Dublin, Republic of Ireland
}

Epidemiological evidence indicates that consumption of diets rich in whole grains is associated with a reduced incidence of a number of chronic diseases including heart disease and type 2 diabetes ${ }^{(1)}$. The mechanisms of protection, however, are unclear. Whole grains, particularly the bran and germ fractions of wheat, are rich in the vitamin E isomers $\alpha$ - and $\gamma$-tocopherol, which may protect against free radical damage, and are also good sources of folate, choline and betaine, which act as physiological methyl donors. The aim of the current study was to assess the post-meal uptake of tocopherols and these methyl donors after consumption of wheat-bran and wheat-aleurone test meals.

The study used a randomised cross-over design, with fourteen healthy subjects (seven males seven females, age 21-43 years, BMI $19-28 \mathrm{~kg} / \mathrm{m}^{2}$ ). On three occasions, each 1 week apart, after an overnight fast subjects consumed $50 \mathrm{~g}$ wheat bran (Bühler, Uzwil, Switzerland), $50 \mathrm{~g}$ aleurone-rich bran (Bühler) or a control meal. Meals were balanced for macronutrients and fibre and were boiled with water before serving to improve palatability. Blood samples were taken at baseline and at $0.5,1,2$ and $3 \mathrm{~h}$ post meal. Plasma was analysed for tocopherols (HPLC), folate (microbiological assay), choline and betaine (liquid chromatography-MS/MS). Data were analysed by repeated measures ANOVA.

Compliance with the study protocol was excellent, with volunteers consuming on average 48.8 (SD 4.8) g of the meals. Following consumption of the wheat-bran or aleurone-rich-bran test meal there were no significant increases in plasma $\alpha$-tocopherol $(P=0.885)$, $\gamma$-tocopherol $(P=0.830)$, folate $(P=0.997)$ or choline $(P=0.911)$ as compared with the control meal. However, there were highly significant increases in plasma betaine following consumption of the wheat bran (2-fold rise from baseline) and aleurone-rich-bran meals (2.5-fold rise from baseline; $P<0.001)$.

In conclusion, the results suggest that wheat bran and aleurone are good sources of bioavailable betaine, which might be of importance to health given the ability of dietary betaine to lower plasma homocysteine ${ }^{(2)}$, an independent risk factor for CVD, particularly stroke ${ }^{(3)}$. No changes in concentrations of choline, folate or vitamin $\mathrm{E}$ were evident, perhaps as a result of the relatively low concentrations of these components in the test meals, the composition of the test meals or losses after boiling. Whether the observations of enhanced plasma betaine would be maintained with longer-term consumption of wheat-bran fractions requires further investigation.

This study is financially supported by the European Commission 6th Framework Programme project HEALTHGRAIN (FP6-514006). It reflects the authors' views.

1. McKeown NM, Meigs JB, Liu S, Wilson PW \& Jacques PF (2002) Am J Clin Nutr 76, 390-398.

2. Cho E, Zeisel SH, Jacques P, Selhub J, Dougherty L, Colditz GA \& Willett WC (2006) Am J Clin Nutr 83, 905-911.

3. Wang X, Qin X, Demirtas H, Li J, Mao G, Huo Y, Sun N, Liu L \& Xu X (2007) Lancet 369, 1876-1882. 